\title{
Reimagining measures of spatial access to health care in low- and middle-income countries: using road network analysis to validate self-reported perceptions of geospatial barriers
}

\author{
Lois Park $^{\mathrm{a}, *}$, Amber Birdwell ${ }^{\text {a }}$, Yao-Yi Chiang ${ }^{\mathrm{b}}$ \\ a Spatial Sciences Institute, University of Southern California, LoisPark@usc.edu,Amber Birdwell, Birdwell@usc.edu \\ ${ }^{b}$ University of Minnesota, YaoYi@umn.edu \\ * Corresponding author
}

Keywords: Global Health, Spatial Access, Network Analysis, Self-reported Barriers, Demographic and Health Survey, Service Provision Assessment, OpenStreetMap, OSM, GIS, LMIC, SDGs

\begin{abstract}
:
Among the many barriers to obtaining health care, spatial access is among the most critical, especially in low- and middleincome countries (LMICs). The average distance to a hospital in LMICs is approximately 30 kilometers, compared to around 5 kilometers in high-income countries (Meara et al., 2015). The lack of geographic access to health care can increase the risk of delayed treatment or lack thereof, leading to increased adverse health outcomes, including death. Thus, characterizing geographic access is crucial for accurately identifying spatial barriers and planning for programs that aim to improve the accessibility of health care for the most vulnerable populations.

The Demographic and Health Survey (DHS) is a major source of data for tracking population health in LMICs, with over 400 surveys in 90 countries since the 1980s. The survey module in the DHS for reproductive-aged women asks them to identify factors, including physical distance to facilities, that pose a big barrier to accessing medical care. This study analyses the response of more than 24,000 individuals in 850 sampled clusters from the 2015-16 Malawi DHS. Using the location of 977 health facilities from a census of health facilities (2013-14 Malawi Service Provision Assessment) and road network data from OpenStreetMap, a routing algorithm is implemented to quantify the distance between the DHS clusters and their nearest health facility.

Preliminary results show that perceiving distance as a significant barrier to accessing health care is indicative of living farther from a health facility than those who do not perceive distance to be a big problem. While further study is needed to determine if this relationship holds for other country contexts and across time, this study is the first, to our knowledge, to validate this qualitative measure against a quantitative criterion.

The implications reach far beyond this study of a specific indicator. This study demonstrates an important way in which spatial scientists can influence the global health dialogue. Through a spatial analysis of an otherwise subjective measure, we have now contributed a spatial significance to it. This allows researchers in other disciplines and decision-makers with little training in the geospatial perspective to develop a spatial competence by engaging with an existing, familiar measure that has been reimagined to represent quantitative spatial attributes. While advancing new, spatially explicit indicators in global health, adding spatial value to existing non-spatial measures is also an important strategy that promotes the use and demand for spatial tools in public health.
\end{abstract}

